\title{
Atypical pleuritic chest pain as diagnostic clue for COVID-19: - A Case Report
}

\author{
M PHANI KRISHNA ${ }^{1}$, Neetu Alex ${ }^{1}$, and Srimathy Venkatesh ${ }^{1}$ \\ ${ }^{1}$ Apollo Hospitals Enterprise Ltd
}

June 5, 2020

\begin{abstract}
Most common clinical manifestations of COVID-19 are fever, dry cough, dyspnea, fatigue, myalgia, sore throat, expectoration, hemoptysis, nausea, diarrhoea, abdominal pain and headache. But pleuritic chest pain as lone presenting symptom with COVID-19 is not reported till date.
\end{abstract}

Atypical pleuritic chest pain as diagnostic clue for COVID-19:

- A Case Report

Dr. M Phani Krishna (1) Dr. Neetu Mariam Alex ${ }^{(2)}$ Dr. Srimathy Venkatesh (3)

(1), (2) - DNB Residents, Department of Internal Medicine, Apollo Hospitals, Greams Road, Chennai, Tamil Nadu, India.

(3) - Senior Consultant, Department of Internal Medicine, Apollo Hospitals, Greams Road, Chennai, Tamil Nadu, India

\section{Corresponding Author:}

Dr. M Phani Krishna

3-1-39, Nagendra Nagar,

Nellore -524002 ,

Andhra Pradesh,

India

Abstract: Coronaviruses are well known human pathogens known to cause respiratory illness. In the first week of December 2019, a cluster of cases diagnosed as "pneumonia of unknown aetiology", were reported in Wuhan, Hubei province in China. A novel coronavirus was isolated from these patients and it was named as SARS-CoV-2 and disease as COVID-19. On March 11 th 2020 , COVID-19 was declared as a pandemic by W.H.O.

Most common clinical manifestations of COVID-19 are fever, dry cough, dyspnea, fatigue, myalgia, sore throat, expectoration, hemoptysis, nausea, diarrhoea, abdominal pain and headache. But pleuritic chest pain as lone presenting symptom with COVID-19 is not reported till date. We present a case of 62-year-old gentleman who presented with worsening long standing pleuritic chest pain and was subsequently diagnosed to have COVID-19.

Keywords: SARS-CoV-2, COVID-19, Pleuritic chest pain. 
Key clinical message : Knowledge about rare and unusual presentations of this newly emerging pandemic is very important for clinicians as missing a case may result in further spread of infection. This case highlights the importance of imaging in COVID-19

Background : Coronaviruses are well known human pathogens known to cause respiratory illness. These are largely divided into 4 genera namely $\alpha, \beta, \gamma$, and $\delta$. $\alpha$ coronaviruses like 229E, NL63, OC43 and HKU1 are known to cause seasonal mild respiratory illness where $\beta$ - Coronaviruses like (severe acute respiratory syndrome) SARS CoV-1 and (middle east respiratory syndrome) MERS CoV were responsible for two epidemic outbreaks in 2002-03 and 2012- 13 respectively. In the first week of December 2019, a cluster of cases diagnosed as "pneumonia of unknown aetiology", were reported in Wuhan, Hubei province in China. On January $7^{\text {th }} 2020$, a novel corona virus was isolated. It was named as Severe acute respiratory syndrome corona virus 2 (SARS-CoV-2) and disease was named as corona virus disease-19 (COVID -19) by World health organisation (WHO). On March 11, 2020, COVID-19 was declared as pandemic by WHO. As on 20-05-2020 about 4731458 cases and 316, 169 deaths were reported affecting around 216 countries around the globe ${ }^{(1)}$.

SARS CoV-2 results in milder forms of disease in most of the cases but it has high infectivity. Elderly and people with underlying co-morbidities are at higher risk of critical illness. Most common clinical manifestations of COVID-19 are fever, dry cough, dyspnea, fatigue, myalgia, sore throat, expectoration, hemoptysis, nausea, diarrhoea, abdominal pain and headache ${ }^{(2,3)}$. But pleuritic chest pain as the sole presentation is very rare with COVID-19. We report a case of 62-year-old gentleman who presented with worsening long standing pleuritic chest pain and he was subsequently diagnosed to have COVID-19.

\section{Case report :}

A 62-year-old male has been presented to our emergency department with the complaints of severe chest pain, more on left lateral side for 1 week which got worsened since 1 day. He had no history of fever, cough, sore throat, shortness of breath. No history of sick contact or recent travel. His medical history is significant for diabetes mellitus type 2, systemic hypertension, old cerebrovascular accident and hypothyroidism.

He had sustained a road traffic accident in second week of March 2019 and he was managed conservatively elsewhere. In view of persistent chest pain he was admitted in our hospital. On evaluation, electrocardiogram (ECG), Chest X-RAY PA view and echocardiogram were found to be normal. Computed tomography (CT) scan chest revealed fibrotic parenchymal strands in left lower lobe, pleural tags with thickening in upper lobes; soft tissue and bony thorax did not show any abnormality (Figure: 1). He was managed conservatively and was discharged. Again in the last week of March, he presented to outpatient department with complaints of high grade fever (Temperature - 103), cough and left side chest pain. Repeat chest X-Ray was normal. He was managed with oral antibiotics and recovered completely.

On admission he had pulse rate of $74 / \mathrm{min}$, blood pressure $110 / 70 \mathrm{~mm}$ of $\mathrm{hg}$, and he was mildly hypoxic requiring 2 litres/min of oxygen via nasal prongs. Systemic examination was unremarkable. A 12-lead ECG showed normal sinus rhythm, no significant ST-T changes. Troponin I was within normal limits. The patient's full blood counts demonstrated normal leucocyte count $\left(8.6 \times 10^{9} / \mathrm{L}\right)$ with mild eosinophilia, monocytosis ( Neutrophils-45\%, lymphocytes- : $34 \%$, eosinophils $-9 \%$, monocytes $-12 \%$ ), haemoglobin of 113 $\mathrm{gm} /$ lit and platelets of $3.43 \times 10^{9} / \mathrm{L}$. Baseline biochemical parameters were within normal limits.

The patient underwent CT-scan chest on the same day and it revealed patchy ground glass opacities predominantly peripheral in both lungs involving all lobes with sub pleural reticulation in both lower lobe (Figure: 2). Nasopharyngeal swab was sent for COVID-19 testing by RT-PCR and it was reported equivocal. Given the current prevalence of COVID-19 and imaging highly suggestive of viral aetiology, we shifted the patient to an isolation room and proceeded with repeat testing which turned out to be positive. He was shifted to designated COVID-19 hospital (Part of our network) and was continued on supportive care and empirical antibiotics (Inj. Ceftriaxone 2gm IV OD and T. DOXY 100mg PO BD). Inflammatory markers like ferritin, $\mathrm{C}$ - reactive protein, and lactate dehydrogenase were all within normal limits and but D-Dimer levels were mildly elevated $(618 \mathrm{ng} / \mathrm{ml})$. Vitamin D levels (20) were low and started on supplements. Blood culture, spu- 
tum gram staining and cultures did not show any significant growth. Sputum X-PERT MTB was negative. Antibiotics were withheld and with appropriate measures his oxygen requirement came down.

With signs of clinical improvement repeat testing done $5^{\text {th }}$ day of illness was negative. But the test repeated after 24 hours again turned out to be positive. Though patient was clinically stable, as per local guidelines he needs to come out negative in two tests 24 hours apart. Repeat testing done on $10^{\text {th }}$ and $11^{\text {th }}$ day of illness were negative. He was discharged and was reviewed a week ago as outpatient, his chest pain subsided and he is doing perfectly well.

\section{Discussion :}

SARS CoV-2 is an enveloped positive sense single stranded RNA virus. It is spherical in shape with spikes projecting from the surface of its envelope giving it a crown like appearance, hence the name coronavirus. The structural proteins include Spike(S), envelope (E), transmembrane (M), nucleocapsid (N). Spike protein is an important determinant of virus entry into host cells and nucleocapsid plays an important role in pathogenesis, replication and RNA packing (4). Incubation period of COVID-19 ranges between 2-14 days with median of 5.2 days ${ }^{(5)}$. Men are affected more and median age is 57 years which is consistent with our patient (A gentleman above 60 years of age). The risk for severe disease increases by $58 \%$ for every 10 years increase in age ${ }^{(2)}$. Most of the clinical features are non-specific and may not reliably establish the diagnosis of COVID-19.

Classical presentation is fever, cough with dyspnea and bilateral chest infiltrates. Fever was seen in $92 \%$ of patients, cough in $70-75 \%$, fatigue in $75 \%$, and chest tightness in $49-62 \%$ of patients ${ }^{(2,3)}$. Other reported symptoms were anorexia, myalgia, sputum production, rhinorrhea, and headache. Altered smell or taste can be the first apparent manifestation in mild category of patients and it was seen in around $64 \%$ of patients ${ }^{(6)}$. Ocular manifestation like increased secretions and conjunctival hyperemia were reported. SARS-CoV-2 viral RNA was detected in tears ${ }^{(7)}$. Gastrointestinal symptoms like diarrhea can be the presenting manifestation in COVID-19.

Though viral pneumonias are associated with pleurisy, it has not been reported till date in association with SARS-CoV-2. After extensive review of literature we did not find a case of COVID-19 with pleuritic chest pain alone as presenting manifestation. In fact it is CT-Scan chest which helped us in arriving at a diagnosis, which explains the importance of imaging in COVID-19. In a study done by Bai HX et al., radiologists in China and the United States were able to distinguish COVID-19 from viral pneumonia on chest CT with high specificity but moderate sensitivity ${ }^{(8)}$. The peripheral lung involvement on CT chest with the background of road traffic accident may explain the worsening pleuritic chest pain in our patient.

Another interesting learning point was about RT-PCR interpretation. In our case initial test was reported equivocal. But chest imaging being highly suggestive of viral pneumonia, we repeated RT-PCR test next day and it turned out to be positive. He improved well with supportive measures and repeat testing done on $5^{\text {th }}$ day was negative. But surprisingly on $6^{\text {th }}$ day it turned out to be positive again. Respiratory isolation measures were continued along with supportive measures and two consecutive tests done on $10^{\text {th }}$ and $11^{\text {th }}$ day were reported negative. All the inflammatory markers like CRP, ferritin, and LDH were within normal limits and D-dimer levels being not significantly elevated would explain the milder form of disease and favorable outcome in our case. He was reviewed as outpatient 2 weeks later and he was doing well.

Conclusion : Key steps to contain the spread of infection include active case finding and isolation of positive cases, contact tracing and quarantine, physical distancing of at least 6 feet at public places and cough etiquette. Knowledge about rare and unusual presentations of this newly emerging pandemic is very important for clinicians as missing a case may result in further spread of infection. This case highlights the importance of chest imaging findings in diagnosis and importance of being vigilant about atypical presentations of COVID-19.

Conflicts of interest : Nil

Funding : Nil 
Author contribution : All authors contributed equally

\section{References :}

1. Corona virus Disease 2019 (COVID-19) Situation Report - 120. World Health Organization. Published May 19, 2020. Accessed May 20, 2020. https://www.who.int/docs/defaultsource/coronaviruse/situation-reports/20200519-covid-19-sitrep-120.pdf?sfvrsn=515cabfb_2

2. Wang et al., (In Press) "Clinical Course and Outcomes of 344 Intensive Care Patients with COVID-19. Am J Respir Crit Care Med. 2020 Apr 8. doi: 10.1164/rccm.202003-0736LE

3. Chen Tao, Wu Di, Chen Huilong, Yan Weiming, Yang Danlei, Chen Guang et al. "Clinical characteristics of 113 deceased patients with coronavirus disease 2019: retrospective study" BMJ 2020;368 :m109

4. X. Li et al., "Molecular immune pathogenesis and diagnosis of COVID-19" Journal of Pharmaceutical Analysis 10 (2020) 102-108.

5. Lauer SA, Grantz KH, Bi Q, et al. The Incubation Period of Coronavirus Disease 2019 (COVID-19) From Publicly Reported Confirmed Cases: Estimation and Application [published online ahead of print, 2020 Mar 10]. Ann Intern Med . 2020; M20-0504. doi:10.7326/M20-0504

6. Spinato G, Fabbris C, Polesel J, et al. Alterations in Smell or Taste in Mildly Symptomatic Outpatients with SARS-CoV-2 Infection.JAMA. Published online April 22, 2020. doi:10.1001/jama.2020.6771

7. Vetter Pauline, Vu Diem Lan, L'Huillier Arnaud G, Schibler Manuel, Kaiser Laurent, Jacquerioz Frederique et al. "Clinical features of covid-19" BMJ 2020; 369 :m1470

8. Bai HX et al., "Performance of radiologists in differentiating COVID-19 from viral pneumonia on chest CT" Radiology. 2020 Mar 10:200823. doi: 10.1148/radiol.2020200823. [Epub ahead of print]

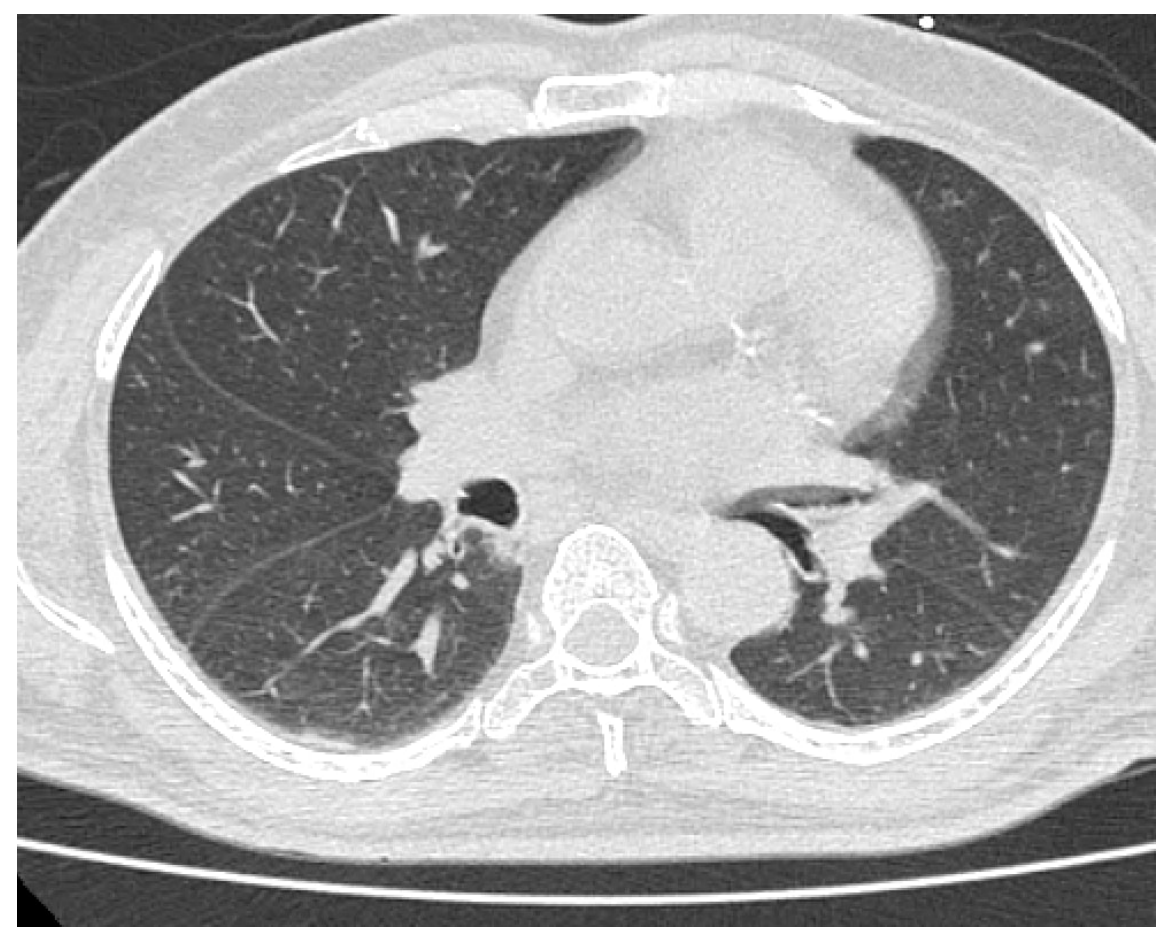




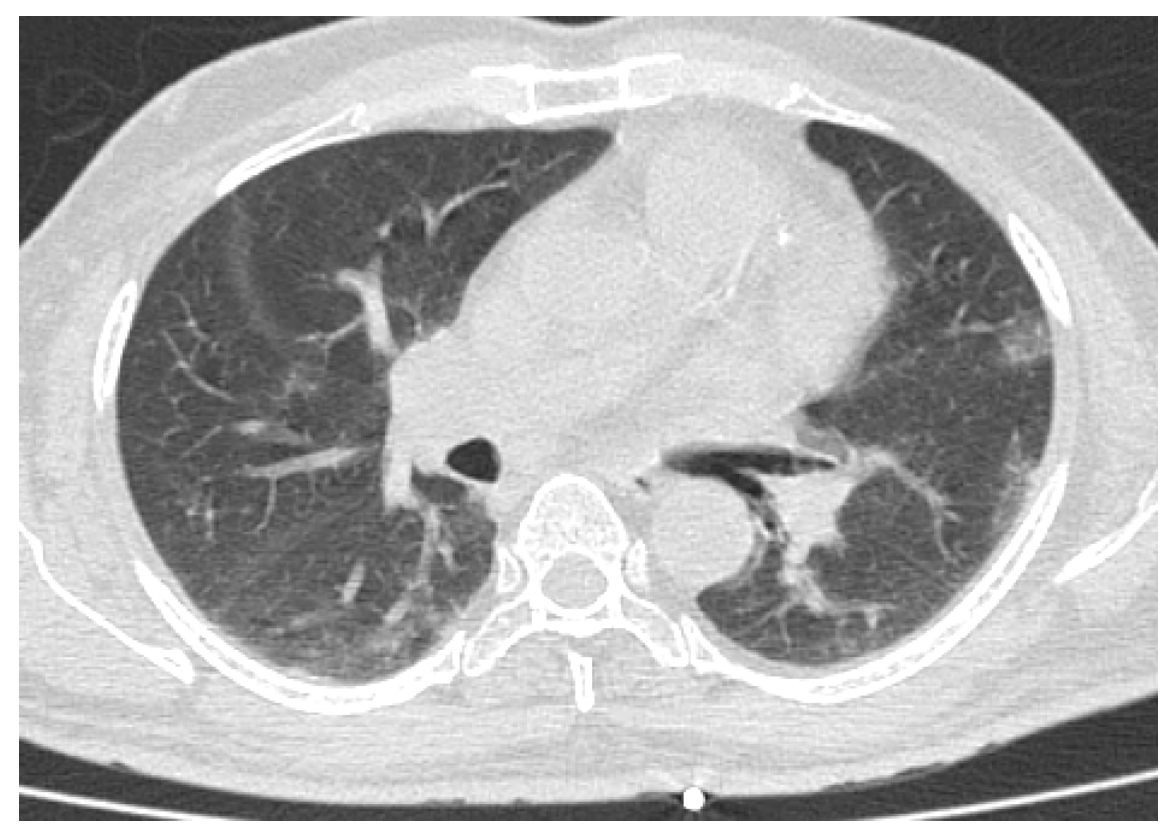

